\title{
EXPERIMENTAL AND NUMERICAL STUDIES OF NONEQUILIBRIUM EFFECTS IN FRAMEWORK OF MAGNETOHYDRODYNAMIC PARACHUTE CONCEPT
}

\author{
V. A. Bityurin, A. N. Bocharov, N. A. Popov, \\ and D.S. Baranov
}

Joint Institute for High Temperature

Russian Academy of Sciences

Moscow, Russia

The magnetohydrodynamic (MHD) parachute effect is studied numerically for the reentry conditions. The effects of the extra ionization due to induced electric field $U \times B$ are taken into account. The efficiency of MHD interaction in terms of drag is estimated. For the flight conditions under consideration, the increase of total drag due to electromagnetic one is more than twice.

\section{INTRODUCTION}

The interest to the supersonic flow control by means of the electromagnetic field has been renewed after papers $[1,2]$, in which several new fields have been proposed. These proposals could be conditionally classified as $(i)$ the flow control and (ii) the power generation. As far as the flow control is concerned, the intensive studies on the influence of magnetic field on the reentry flight were carried out in 1990s and early 2000s [3-6]. It was found that magnetic field can significantly change the position and the shape of the bow shock, thus changing the aerodynamics of the reentry vehicle. The most important practical conclusion made in these studies is that the mitigation of the heat flux on the vehicle surface could be significant.

As far as the power generation is concerned, most studies were carried out within the "AJAX" concept first presented in [7]. The less studied is the "dream" concept of on-board MHD generator [1, 8-10]. Earlier studies have shown the principal possibility to extract $\sim 1 \mathrm{MW}$ electric power from the hypersonic airflow. Later, in [11], the on-board MHD generation was studied for the reentry

This is an Open Access article distributed under the terms of the Creative Commons Attribution-Noncommercial License 3.0, which permits unrestricted use, distribution, and reproduction in any noncommercial medium, provided the original work is properly cited. 


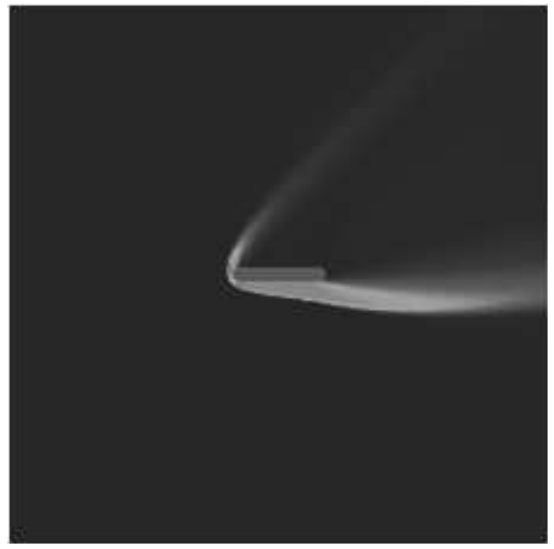

(a)

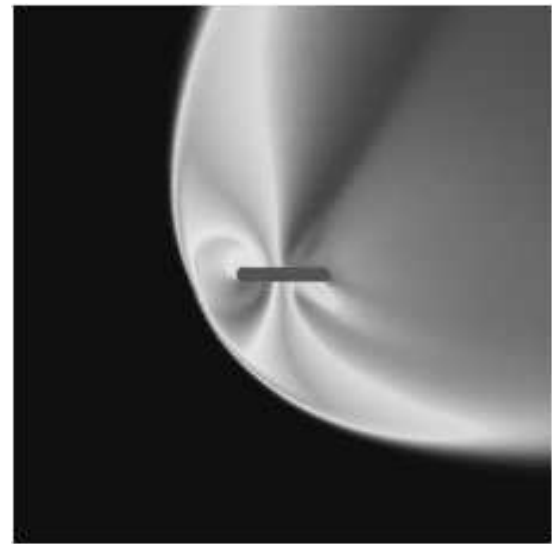

(b)

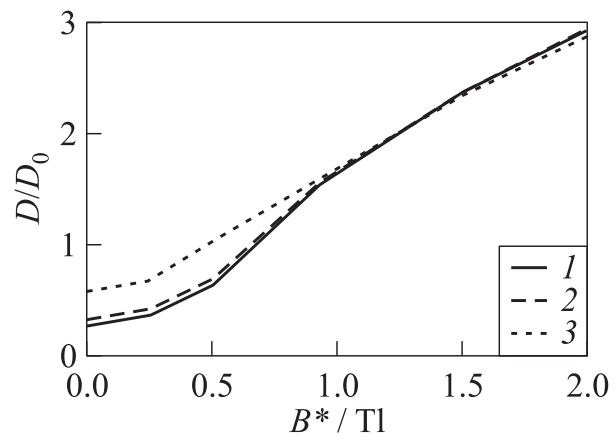

(c)

Figure 1 Performance of MHD parachute for experimental conditions: $(a)$ original (no MHD) pressure field; $(b)$ pressure field at magnetic field $B^{*}=2 \mathrm{Tl}$; and $(c)$ electromagnetic drag vs. magnetic field $\left(1-\alpha=0^{\circ} ; 2-10^{\circ}\right.$; and $\left.3-\alpha=30^{\circ}\right)$. Flow conditions (the TsAGI hypervelocity MHD facility $[2,10]): V=5000 \mathrm{~m} / \mathrm{s}, P=30 \mathrm{~Pa}$, $\mathrm{M}=9.7$, angle-of-attack $=30^{\circ}$, length of magnet $96 \mathrm{~mm}$, and diameter of conductors $15 \mathrm{~mm}$

conditions. Just recently [10], the experimental demonstration of the hypersonic MHD generation was presented.

As a consequence of the power generation studies, the novel concept of the MHD break (MHD parachute) has been proposed in [8-10]. Figure 1 demonstrates the performance of the MHD parachute under experimental conditions of the hypersonic MHD facility: the total drag can be increased in order of magnitude due to the interaction of electric current in plasma with applied magnetic field. It was also noticed that the electromagnetic drag depends neither on the 


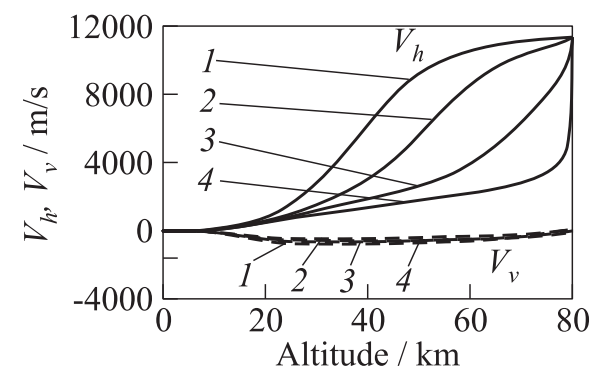

(a)

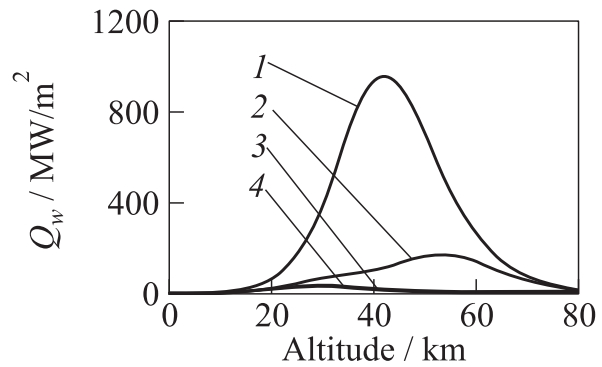

(b)

Figure 2 Magnetohydrodynamic parachute as the means to prevent high heat loads while reentry path: $(a)$ horizontal ( $V_{h}$, solid curves) and vertical $\left(V_{v}\right.$, dashed curves) velocity vs. altitude; $(b)$ peak heat loads vs. altitude: $1-S_{\mathrm{mhd}}=0 ; 2-1 ; 3-10$; and $4-S_{\mathrm{mhd}}=100$. Reentry conditions: altitude $=80 \mathrm{~km}, V_{h}=11.4 \mathrm{~km} / \mathrm{s}, V_{v}=0$, and angle-of-attack $=30^{\circ}$

angle-of-attack, nor on the body shape. Figure 2 demonstrates the main idea of MHD parachute: to prevent rather than to protect. This means that the MHD deceleration of the vehicle should take place at the altitudes where heat fluxes onto vehicle surface are still not high to damage thermal protection system. The flight at lower altitudes (higher gas density) could occur at much lower velocities.

Simulations of the MHD parachute for the real flight conditions $(\mathrm{M}=24)$ showed that the sufficient thermal ionization could take place behind the bow shock only near the critical point of the vehicle. The downstream flow is chemically frozen, and the charged particles are only those produced in the leading part of the shock layer. The ionization degree is not high enough to produce the significant MHD interaction in the flow. At the same time, it has been noticed that the reduced electric field $|\mathbf{U} \times \mathbf{B}| / n$ ( $\mathbf{U}$ is the velocity; $\mathbf{B}$ is the magnetic flux density; and $n$ is the total number density) can be as high as $10^{3}$ Townsend, which is much higher than breakdown field in air. A natural idea to take into account the ionization due to a strong electric field has been first considered in $[12,13]$. It has been found there that the ionization degree can be increased in two orders of magnitude in the flow over the magnet.

In the current paper, the MHD parachute effects are demonstrated in the hypersonic flow of nitrogen over the thick plate with magnet built into plate body. The improved model of the "electric field kinetics" is considered.

\section{PROBLEM SETUP}

Consider the supersonic flow over a thick plate with the cylindrical leading part (Fig. 3). The free-stream flow conditions are as follows: gas is nitrogen, the 


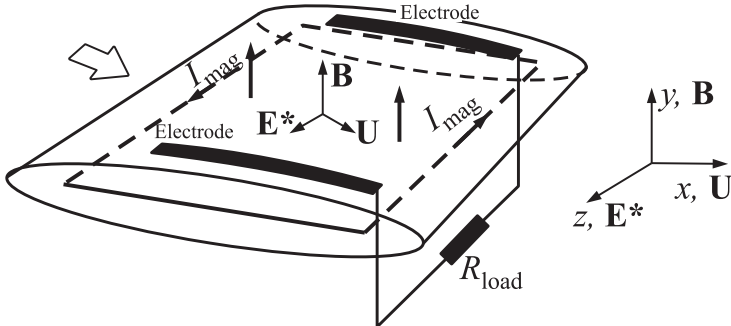

(a)

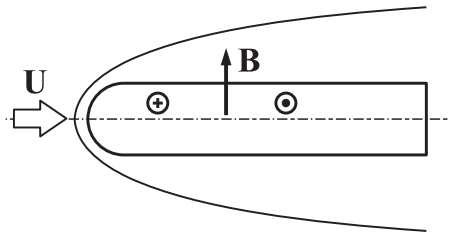

(b)

Figure 3 Layout of on-board MHD generator $(a)$ and layout of computational domain in $x y$ plane $(b)$

velocity is $11137 \mathrm{~m} / \mathrm{s}$, the static pressure is $16.6 \mathrm{~Pa}$, the gas density is 2.34 $\times 10^{-4} \mathrm{~kg} / \mathrm{m}^{3}$, the static temperature is $238.5 \mathrm{~K}$, and the Mach number is 35.2 . The plate length along the flow direction is $0.7 \mathrm{~m}$, the radius of cylindrical part of the plate is $0.016 \mathrm{~m}$. Magnetic field is generated by the wire with current; so, it is normal to the plate surface over the most part of the plate. Considering the plate long enough in the direction normal to the picture (z-direction), one can assume that magnetic field is created by two conductors with opposite currents parallel to $z$-direction. So, the magnetic field is two-dimensional (2D) $\mathbf{B}(x, y)$. The distance between the conductors is $0.3 \mathrm{~m}$, the left one is $0.1 \mathrm{~m}$ left from the leading edge of the plate. It is convenient to characterize the magnetic induction as the field value $B^{*}$ on the plate surface just over the conductor. The value of the field in the center of the plate (between two conductors) is approximately $B^{*} / 7$. For zero angle-of-attack, one can consider the flow symmetric respective to axis $y=0$.

The case of so-called short-circuit MHD generator, that is, $E_{z}=-\partial \varphi / \partial z=0$ ( $\varphi$ is the electric potential; and $E_{z}$ is the mean $z$-component of electric field) will be considered. Assuming also that all flow variables do not depend upon $z$-coordinate (near-electrode processes are neglected), the $2 \mathrm{D}$ flow, that is, $\partial / \partial z \approx 0$ for any flow variables will be considered. The Faraday electric current, $j_{z}$, and induced electric field, $E_{z}^{*}=(\mathbf{U} \times \mathbf{B})_{z}$ are the functions only of $(x, y)$. In $2 \mathrm{D}$ problem, one can introduce the electric potential $\varphi(x, y)$, electric field $\mathbf{E}(x, y)=-\nabla \varphi$, and electric current density $\mathbf{j}(x, y)=\left(j_{x}, j_{y}, j_{z}\right)$.

Continuum one-fluid two-temperature model is considered; so, the gas can consist of heavy particles, $\mathrm{N}_{2}, \mathrm{~N}, \mathrm{~N}_{2}^{+}, \mathrm{N}^{+}$, with the same gas temperature and electrons with the electron temperature. The quasi-neutrality is assumed as well the drift-diffusion approach. This allows one to apply the generalized Ohm's law along with the steady equation for electric charge conservation. The key point of the model is a treatment of reaction rates. In this study, traditional approach is considered. That is, the rates of the electron impact ionization 
reactions depend directly on the reduced electric field, $E / n$ ( $E$ is the absolute value of the electric field strength, and $n$ is the total number density). Another approach in which the effective ionizing electric field depends on the magnetic field is planned to be considered later. In estimating transport properties of the mixture, it is assumed that the collision frequencies of the processes with participating of electrons are determined by the electron temperature. The latter is found from the corresponding transport equation. In the equation, both the elastic and inelastic losses are estimated, the energy gain from the electric field is accounted for, and the anisotropic electron heat conductance is considered. The inelastic losses model was designed on the base of papers [14-16].

\section{GOVERNING EQUATIONS}

Under assumptions made above, the following set of $2 \mathrm{D}$ equations is considered:

$$
\begin{gathered}
\frac{\partial \rho}{\partial t}+\nabla(\rho \mathbf{U})=0 \\
\frac{\partial \rho \mathbf{U}}{\partial t}+\nabla(\rho \mathbf{U} \cdot \mathbf{U})+\nabla \tau=-\frac{\partial P}{\partial \mathbf{r}}+\mathbf{j} \times \mathbf{B} ; \\
\left.\frac{\partial \rho e^{0}}{\partial t}+\nabla\left(\rho e^{0}+P\right) \mathbf{U}\right)+\nabla \mathbf{q}+\nabla(\mathbf{U} \tau)=\mathbf{j} \mathbf{E} ; \\
\left.\frac{\partial \rho e_{e}}{\partial t}+\nabla\left(\rho e_{e}+P_{e}\right) \mathbf{U}\right)+\nabla \mathbf{q}_{e}=\mathbf{j} \mathbf{E}^{*}+Q_{e e}-Q_{e i} ; \\
\frac{\partial \rho_{i}}{\partial t}+\nabla\left(\rho_{i} \cdot \mathbf{U}\right)+\nabla \Gamma_{i}=\omega_{i} ; \\
\nabla \mathbf{j}=0, \quad \mathbf{j}+\frac{\beta}{B}(\mathbf{j} \times \mathbf{B})=\sigma\left(\mathbf{E}+(\mathbf{U} \times \mathbf{B})+\frac{\nabla P_{e}}{e n_{e}}\right), \quad \mathbf{E}=-\frac{\partial \varphi}{\partial \mathbf{r}} \\
\frac{\partial}{\partial \mathbf{r}}=\mathbf{e}_{x} \frac{\partial}{\partial x}+\mathbf{e}_{y} \frac{\partial}{\partial y}, \nabla=\frac{\partial}{\partial x}+\frac{1}{y^{\xi}} \frac{\partial}{\partial y} y^{\xi}, \rho=\sum \rho_{i} .
\end{gathered}
$$

Here, $\mathbf{j}$ is the electric current density; $\mathbf{E}$ is the electric field strength; $\varphi$ is the electric potential; $\mathbf{E}^{*}=\mathbf{E}+\mathbf{U} \times \mathbf{B}$ is the electric field in the coordinate system moving with the flow; $\sigma$ is the scalar electric conductivity; $\mathbf{B}$ is the magnetic flux density; $\beta$ is the Hall parameter; $\rho_{i}$ are the partial densities; $\rho$ is the total fluid density; $\mathbf{U}=\left(U_{x} U_{y}\right)$ is the mass-averaged flow velocity; $P$ is the gas-kinetic pressure; $e^{0}$ is the total specific energy; $e_{e}=(3 / 2) Y_{e} R_{e} T_{e}$ is the specific electron energy; $\mathbf{e}_{x}, \mathbf{e}_{y}$ are the unit base vectors; and $\xi=0$ for cartesian coordinate system. The equations of state for the fluid are specified as follows: 


$$
\begin{gathered}
e^{0}=e+\frac{U^{2}}{2} ; \quad e=h-\frac{P}{\rho} ; \\
h=\sum Y_{i} h_{i}, h_{i}\left(T_{i}\right)=h_{i, f}+\int_{T_{\mathrm{ref}}}^{T_{i}} C_{p i}(T) d T ; \\
P=\sum P_{i} ; \quad P_{i}=\rho_{i} R_{i} T_{i} ; \quad R_{i}=\frac{R^{0}}{W_{i}} .
\end{gathered}
$$

Here, $e$ is the specific internal energy; $h$ is the specific internal enthalpy; $h_{i}$ is the partial specific internal enthalpy; $Y_{i}=\rho_{i} / \rho$ is the mass fraction of the $i$ th component; $h_{i, f}$ is the formation enthalpy of the $i$ th component; and $C_{p i}(T)$ is the $i$ th heat-capacity at constant pressure. These are approximated by functions of temperatures. For all molecules, atoms and ions $T_{i}=T ; T_{e}$ is the electron temperature. The terms $Q_{e e}$ and $Q_{e i}$ are the elastic and inelastic losses, respectively. The inelastic losses include the energy loss to ionization and dissociation due to electron impact as well as the losses to the excitation of internal energy states of $\mathrm{N}_{2}$ and $\mathrm{N}$.

Mass diffusion flux $\Gamma_{i}$, total heat flux $\mathbf{q}$, and electron heat flux $\mathbf{q}_{e}$ are specified as

$$
\begin{gathered}
\Gamma_{i}=-\rho D_{i} \frac{\partial Y_{i}}{\partial \mathbf{r}} ; \quad \mathbf{q}=-\lambda \frac{\partial T}{\partial \mathbf{r}}+\sum_{i \neq e} h_{i} \Gamma_{i}+\mathbf{q}_{e} ; \\
\mathbf{q}_{e}+\frac{\beta}{B} \mathbf{q}_{e} \times \mathbf{B}=-\lambda_{e} \nabla T_{e}-\frac{5}{2} k T_{e} \frac{1}{e} \mathbf{j} .
\end{gathered}
$$

Here, $D_{i}$ is the diffusion coefficient of the $i$ th component; $\lambda$ is the mixture heat conductivity coefficient; $\lambda_{e}$ is the electron heat conductivity; and

$$
\lambda_{e}=\frac{n_{e} k^{2} T_{e}}{m_{e} \nu_{e}}
$$

where $n_{e}$ is the electron number density, $m_{e}$ is the electron mass, $\nu_{e}$ is the mean electron collision frequency, and $k$ is the Boltzman constant.

Viscous stress tensor is:

$$
\tau_{i j}=\frac{2}{3} \eta \delta_{i j} \nabla \mathbf{U}-\eta \frac{\partial u_{i}}{\partial x_{j}}
$$

where $\delta_{i j}$ is the Kronecker symbol.

Coefficients $D_{i}, \lambda$, and $\mu$ were determined in accordance with $[17,18]$. Conductivity was determined as follows:

$$
\begin{gathered}
\sigma=e n_{e} \mu_{e} ; \quad \mu_{e}=\frac{\sigma}{e n_{e}}=e \frac{\tau_{e}}{m_{e}} \equiv \frac{\beta_{e}}{B} ; \quad \tau_{e}=\nu_{e}^{-1} ; \\
\nu_{e}=\sum \nu_{e j}, \nu_{e j}=n_{j} u_{e} Q_{e j} .
\end{gathered}
$$


Here, $e$ is the electron charge; $\mu_{e}$ is the electron mobility; $\nu_{e}$ is the mean electron collision frequency; $n_{j}$ is the number density of particles of the $j$ th kind; $u_{e}$ is the mean thermal electron velocity; and $Q_{e j}$ is the collision cross section for collisions of electrons with particles of the $j$ th kind.

\section{CHEMICAL KINETICS MODEL}

The production rate of the $i$ th chemical component due to chemical reactions is given as:

$$
\begin{gathered}
\omega_{i}=W_{i} \sum_{r=1}^{N_{r}}\left[\nu_{i, r}^{\prime \prime}-\nu_{i, r}^{\prime}\right]\left(k_{\mathrm{fr}} \prod_{l=1}^{N} c_{l}^{\nu_{l, r}^{\prime}}-k_{\mathrm{br}} \prod_{l=1}^{N} c_{l}^{\nu_{l, r}^{\prime \prime}}\right) ; \\
c_{i}=\frac{\rho_{i}}{W_{i}} ; \quad k_{\mathrm{fr}, \mathrm{br}}=a T_{*}^{b} \exp \left(-\frac{E_{a}}{T_{*}}\right) .
\end{gathered}
$$

Here, $c_{i}$ is the molar concentration of the $i$ th component; $W_{i}$ is its molecular mass; $\nu_{l, r}^{\prime}$ and $\nu_{l, r}^{\prime \prime}$ are the stoichiometric coefficients of reagents and products in the $r$ th reaction; $k_{\mathrm{fr}}$ and $k_{\mathrm{br}}$ are the forward and backward reaction rate constants, respectively; $a, b, E_{a}$ are the reaction parameters; $N_{r}$ is the total number of reactions; and $N$ is the total number of components. For thermal kinetics, $E_{a}$ is the activation energy and $T^{*}=T$. For "electric field kinetics," $T^{*}=E / n_{0}$ is the value of reduced electric field, and $E_{a}$ is the characteristic reduced electric field. For "field" recombination reactions, $T^{*}=T_{e}$.

The thermal kinetics scheme consists of 14 reactions. "Field" kinetics consists of electron impact reactions for $\mathrm{N}_{2}$ ionization and dissociation, $N$ ionization, and backward recombination reactions determined by the electron temperature. "Field" reaction rate constants, as well as the term $Q_{e i}$ were calculated from the solution of Boltzman equation and approximated as functions of reduced electric field and electron temperature.

In the previous work [13], the authors assumed that magnetic field does not affect directly on the field kinetics. However, in [19] it is shown that electron energy distribution function (EEDF) should depend on the magnetic field. Therefore, here the approximation to the reduced electric field calculated in [19] will be used:

$$
Y=0.579 X^{0.62}
$$

where $Y=E / n_{0} \cdot 10^{16} \mathrm{~V} \cdot \mathrm{cm}^{2}$, and $X=\left(\mathbf{J E}^{*}\right) /\left(e n_{e} n_{0}\right) \cdot 10^{10} \mathrm{~V} \cdot \mathrm{cm}^{3} / \mathrm{s}$.

Actually, relation (7) means that effective reduced electric field participating in ionization kinetics diminishes due to Hall effect. 


\section{INITIAL AND BOUNDARY CONDITIONS}

To solve the set of partial differential equations (1)-(6) the following initial and boundary conditions are specified for flow variables. Initial solution is those obtained at $B^{*}=0$ (see Fig. $3 b$ ). On the left boundary, free-stream flow conditions are set, and chemical composition is specified as follows: $\rho_{\mathrm{N}_{2}} / \rho=1, \rho_{j}=0$, $j=2, \ldots, 5$. For electric potential and electron temperature, the "outflow" conditions are set: $\left(\mathbf{n}_{g} \nabla \varphi\right)=0$. Here, $\mathbf{n}_{g}$ is the unit normal vector. On the right boundary, outflow conditions are set for all variables. The plate surface is considered as noncatalytic and nonconductive, with fixed wall temperature: $T_{w}=1800 \mathrm{~K},\left(\mathbf{n}_{g} \nabla \rho_{j}\right)=0,\left(\mathbf{n}_{g} \mathbf{j}\right)=0, \mathbf{U}_{w}=0$. Part of the boundary between the left boundary and the plate is considered as symmetry axis. Typically, calculations were carried out such that characteristic magnetic field increases from smaller to larger value with the rate $1 \mathrm{Tl} / \mathrm{ms}$ and holds then constant.

\section{COMPUTATIONAL DETAILS}

The problem has been solved in the computational domain consisting of the 160 grid cells along the body and 120 cells in the direction normal to it (C-type grid has been used). Strong stretching of grid-nodes has been applied near the body wall in the normal direction. Grid-independence gas been tested for no MHD case on the grid containing 240 cells in the normal direction. Inviscid fluxes are computed with AUSM-type flux-splitting technique, and viscous fluxes are computed using central differences. All calculations have been done on PC (Intel Core 2 Duo) using sequential code. Typically, from 18 to $36 \mathrm{~h}$ were required to obtain each steady-state solution. However, several cases were running simultaneously.

\section{RESULTS}

Free-stream flow conditions correspond to one of the trajectory point of Stardust spacecraft entry flight path (see, for example, [20]). Original flow field is presented in Fig. 4. The gas temperature behind the bow shock at the stagnation line is about $23,000 \mathrm{~K}$, pressure is $28 \mathrm{kPa}$, electron number density is about $2.3 \cdot 10^{15} \mathrm{~cm}^{-3}$, which corresponds to $1.4 \%$ of relative concentration (mole fraction). Original drag for this configuration is $330 \mathrm{~N} / \mathrm{m}$ (viscous component is about $35 \mathrm{~N} / \mathrm{m}$ ).

Figure 5 represents the same distributions for the reference magnetic induction $B^{*}=1.8 \mathrm{Tl}(0.25 \mathrm{Tl}$ at central point on the surface between two conductors shown in figures). Interaction zone size is comparable with the magnetic field 


\section{Temperature / K}

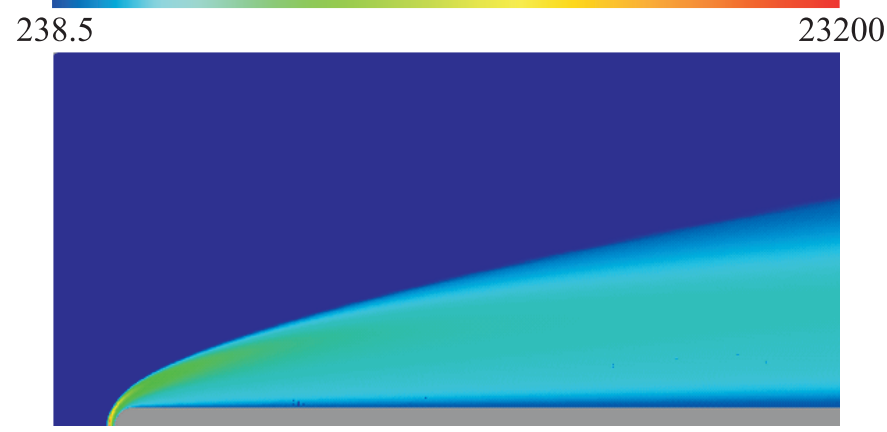

(a)

Pressure / bar

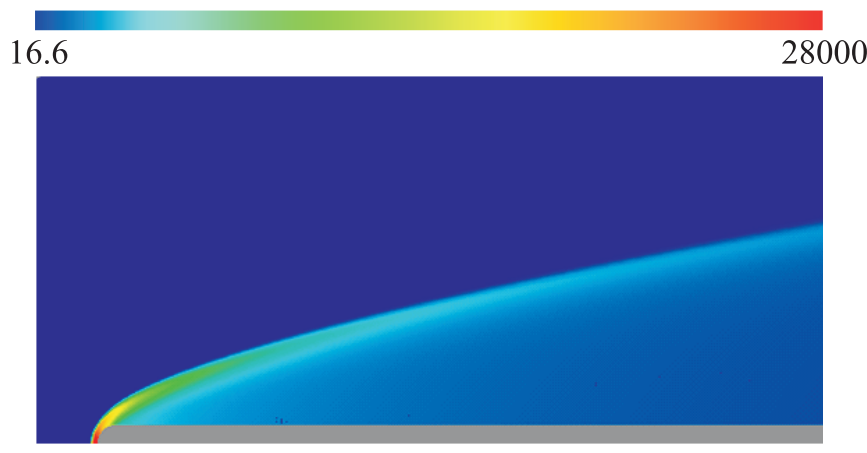

(b)

Electron number density

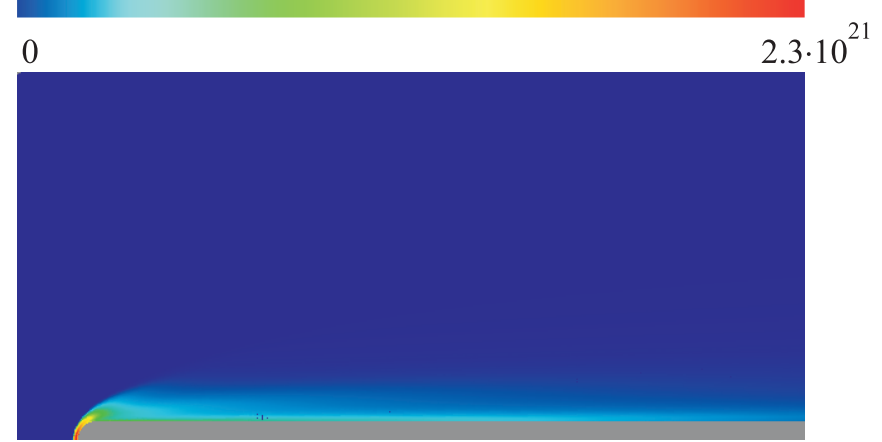

(c)

Figure 4 Temperature $(a)$, pressure $(b)$, and electron number density $(c)$. No magnetic field case. (Refer Bityurin et al., p. 537.) 
Temperature / K

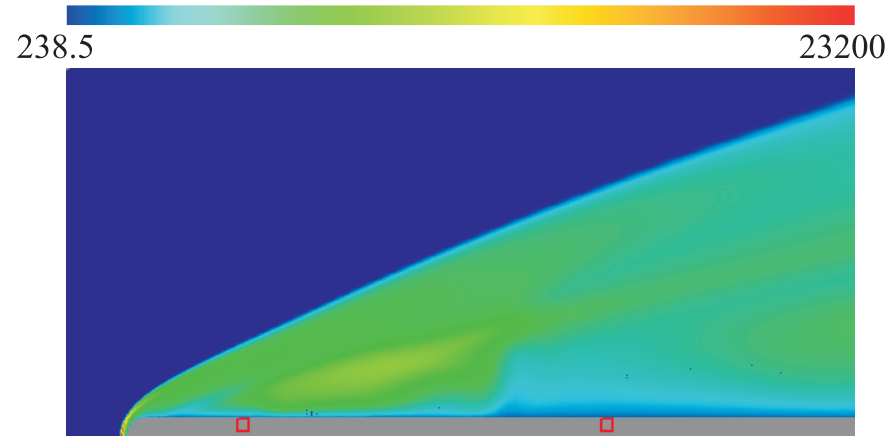

(a)

Pressure / bar

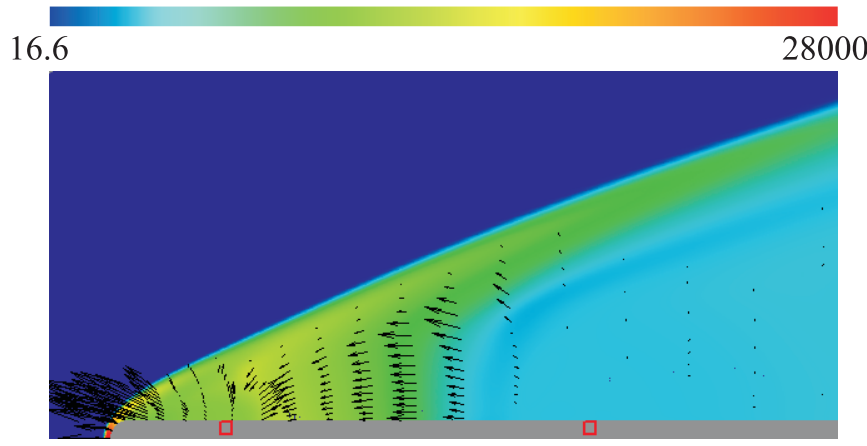

(b)

Electron number density

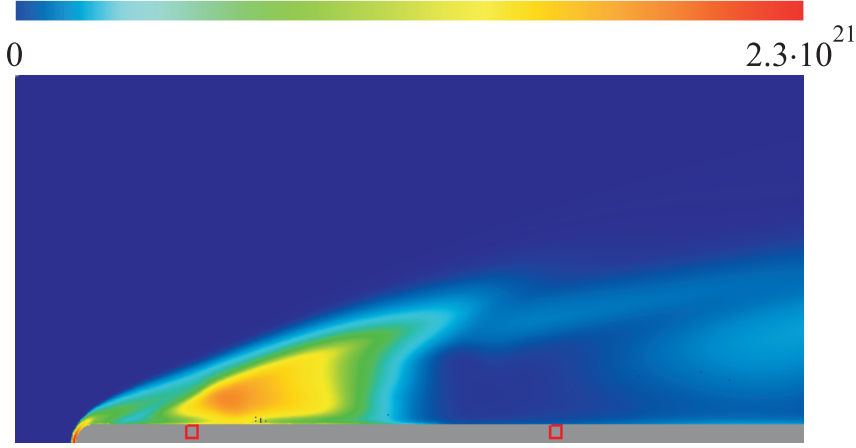

(c)

Figure 5 Temperature $(a)$, pressure and force $(b)$, and electron number density $(c)$; $B^{*}=1.8 \mathrm{Tl}$. (Refer Bityurin et al., p. 538.) 
Temperature / K

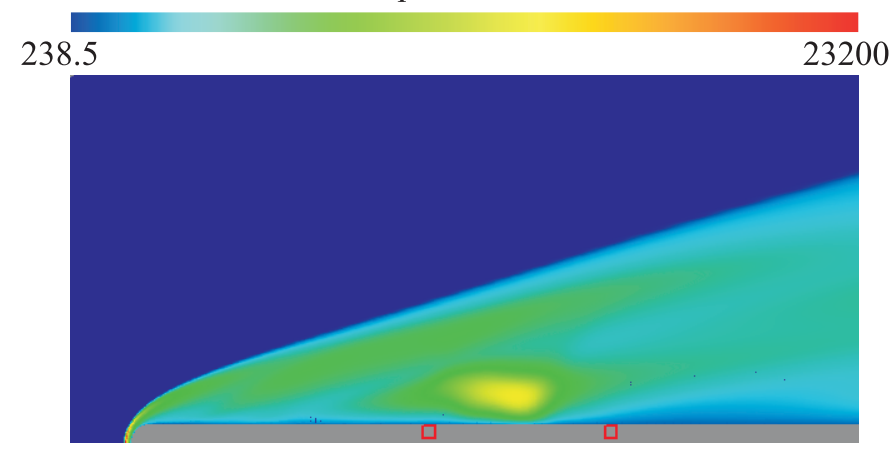

(a)

Pressure / bar

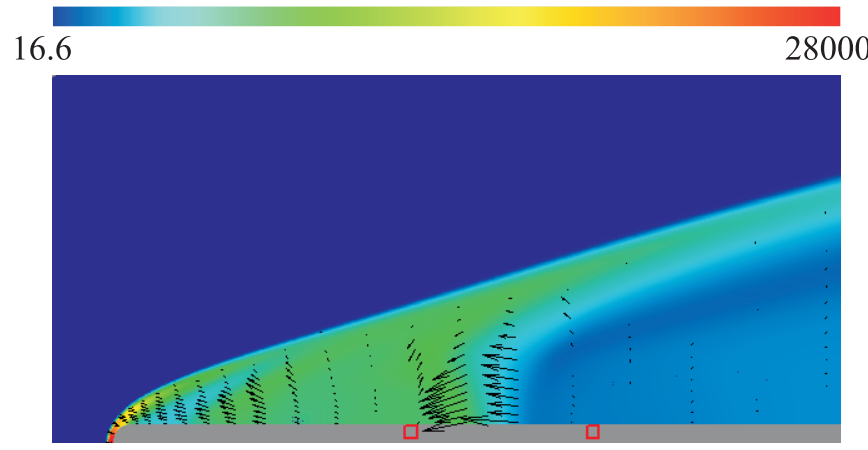

(b)

Electron number density

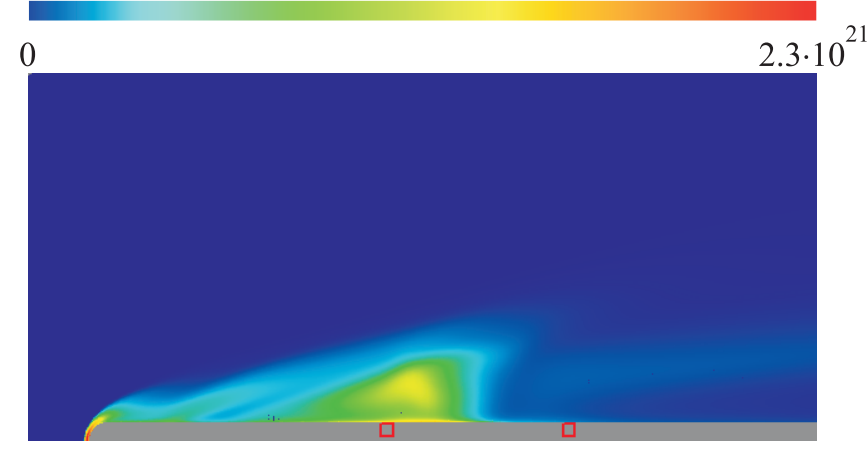

(c)

Figure 6 Temperature $(a)$, pressure and force $(b)$, and electron number density $(c)$; $B^{*}=1.8 \mathrm{Tl}$, shorter magnetic base size.(Refer Bityurin et al., p. 539.) 
base $0.3 \mathrm{~m}$. However, main contribution to the drag is made in the leading part of the shock layer (Fig. $5 b$ ). Electromagnetic drag is about $400 \mathrm{~N} / \mathrm{m}$; so, total drag is about $700 \mathrm{~N} / \mathrm{m}$ (viscous component vanishes). The electron concentration reaches $5 \%$ in the interaction zone (Fig. $5 \mathrm{c}$ ). Further increase in magnetic induction to $2.7 \mathrm{Tl}$ results in increase of electromagnetic drag to $540 \mathrm{~N} / \mathrm{m}$.

Another test series were made to "clean" parachute effect and to eliminate the influence of the leading part of shock layer. This was made by decreasing the magnetic field base in two times (Fig. 6). For such configuration, electromagnetic drag is $170 \mathrm{~N} / \mathrm{m}$ that is slightly less than the ratio of magnetic base sizes. As consequence, the increase of angle of oblique shock is less than in the previous case.

In both cases considered here, the intensity of MHD interaction is high, but less than in earlier prediction with seeded flow. Nevertheless, even under nonequilibrium conditions of realistic flight, the parachute effect remains remarkable and suitable for trajectory control.

As far as the nonequilibrium ionization is concerned, the effect turned out rather small except for regions just behind the shock. In these regions, reduced electric field (in the sense of approximation (7) from paper [19]) may be as high as $500 \mathrm{Td}$ while it is about $20-50 \mathrm{Td}$ in the interaction zone. The influence of the nonequilibrium ionization is still not completely clear, especially within the shock structure. It follows from formal estimations that the very high electric field can take place within the shock structure at very moderate values of Hall parameter. Therefore, further studies of the extended nonequilibrium ionization at the presence of magnetic field are needed to understand its influence on the flow.

\section{EXPERIMENTAL TEST ON THE NONEQUILIBRIUM IONIZATION DUE TO MAGNETIC FIELD}

The experimental tests were carried out on the hypersonic MHD Facility [810, 13]. Common view of the model (thick plate) mounted in test section is shown in Fig. $7 a$. Top view of the model is schematically shown in Fig. $7 b$. Also, electrode system and magnetic coil are shown in this figure. More details on the experimental setup can be found in [10]. The difference of current setup from those typically used in the previous works is the absence of MHD accelerator, which provided the hypervelocity flow in the test section (typically, $U \sim 5000-7500 \mathrm{~m} / \mathrm{s}$, Mach number $\sim 8-15$ ).

In the current tests, the following flow conditions were tested. The freestream velocity was estimated as $U \sim 2900 \mathrm{~m} / \mathrm{s}$ (Mach number $\leq 6$ ), static pressure $\sim 80 \mathrm{~Pa}$, amplitude of the 2 -millisecond pulse magnetic field $\sim 1 \mathrm{Tl}$. 


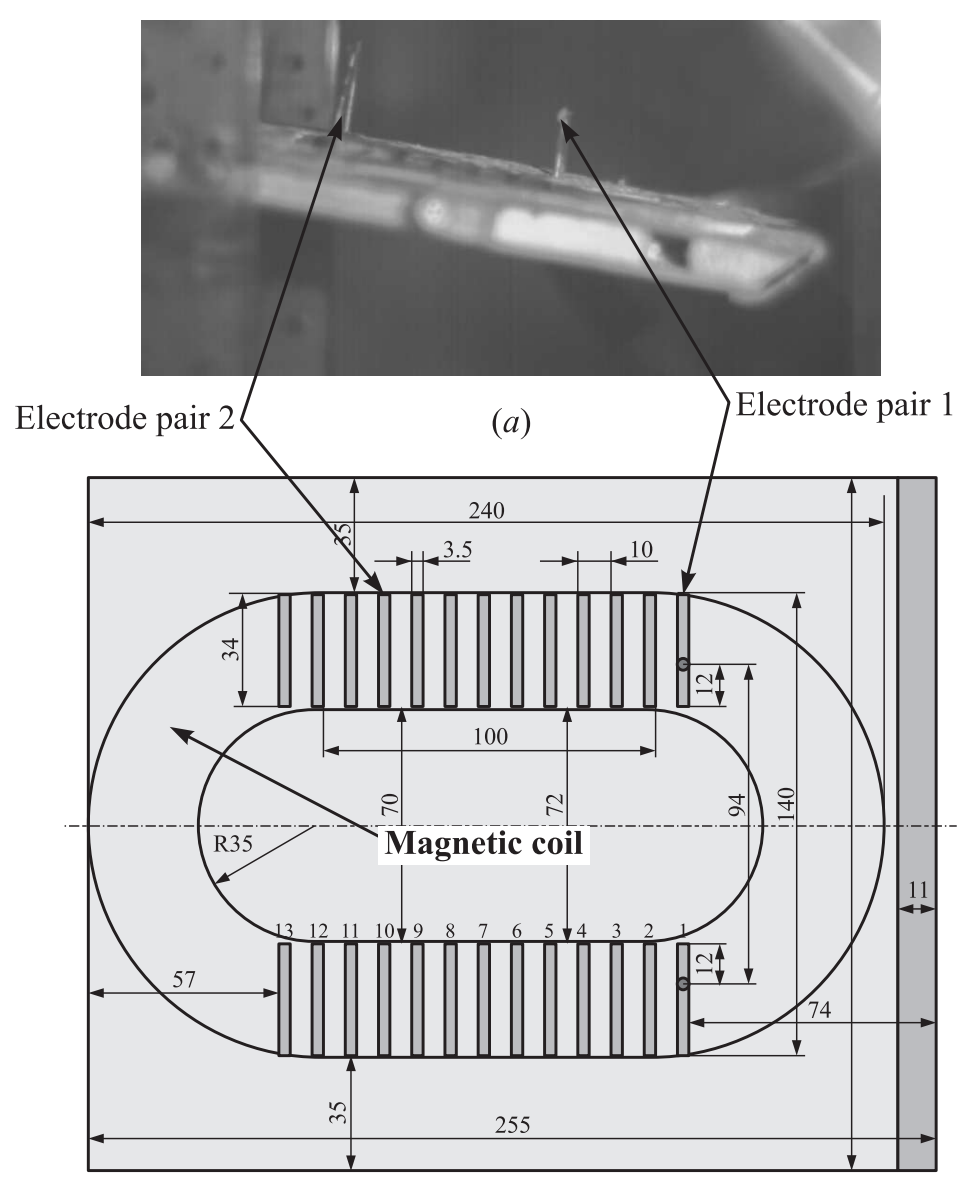

(b)

Figure 7 View of the test plate with the magnetic coil inside mounted in the test section $(a)$ and top view of the plate showing the electrode system and magnetic coil $(b)$. Flow from right to left. Dimensions are in millimeters

Such conditions were selected since no thermal ionization in the shock layer is expected, $T_{\max }<4000 \mathrm{~K}$. According to estimations, the reduced electric field can reach the value of $300 \mathrm{Td}$ under the bottom side of the model, which is high enough for field ionization.

Figure 8 represents two photos of the flow under bottom side of the test plate, in the place where maximal ionization was predicted. The visualization of the flow was done with the camera (Citius, $5000 \mathrm{fps}$ ). Bright strip at the bottom picture points out on the presence of electrons, which might appear 


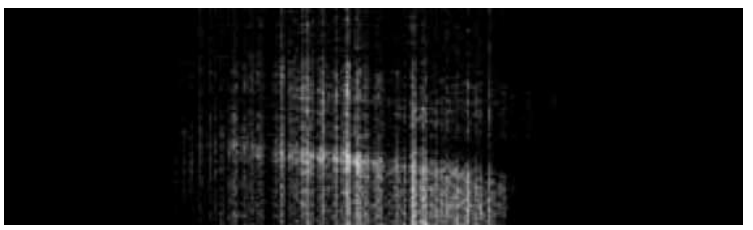

(a)

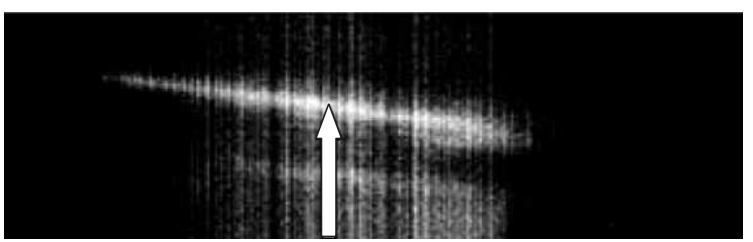

(b)

Figure 8 Visualization of the effect of nonequilibrium ionization in supersonic air flow around a test plate: $(a)$ no magnetic field, and $(b)$ flow with 2-millisecond pulsed magnetic field $\left(B^{*} \sim 1 \mathrm{Tl}\right)$. Flow from right to left, angle-of-attack is $10^{\circ}$

only due induced electric field. No special diagnostic tools were applied on this preliminary stage, only electric potential drop (Faraday voltage) was measured in the circuits designated as electrode-pair-1 and electrode-pair-2 in Fig. 7. Very small signal was registered in the electrode-pair-2 circuit, and Faraday voltage of about $30 \mathrm{~V}$ was detected in electrode-pair-1. In both circuits, the load resistance $66 \mathrm{kOhm}$ was used.

\section{CONCLUDING REMARKS}

This paper continues the studies of MHD flow within the MHD parachute concept. For real flight conditions and magnetic induction level considered, the electromagnetic component of total drag may 1.6 times exceed hydrodynamic one for lab-scale model $(0.3 \mathrm{~m})$. That is, calculations confirm the principal feasibility of entry trajectory control by magnetic field even under nonequilibrium conditions of real flight.

The first experimental tests pointed out on the presence of ionization in the cold air flow due to applied magnetic field. So, further studies of the ionization effect in magnetic field are necessary.

The most recent studies of MHD parachute under real air conditions made for both lab- and space-scale models (see, for example, [21]) revealed that total drag can be increased in almost order of magnitude $\left(B^{*} \sim 1 \mathrm{Tl}\right)$, even though the induced magnetic field deteriorates the MHD interaction. 


\section{ACKNOWLEDGMENTS}

This work was partially supported by the RAS Program \#9 and Russian Foundation for Basic Research (Grant \#08-08-00998a).

\section{REFERENCES}

1. Bityurin, V.A., V.A. Zeigarnik, and A.L. Kuranov. 1996. On a perspective of MHD technology in aerospace applications. AIAA Paper No. 96-2355.

2. Bityurin, V.A., J. T. Lineberry, V. G. Potebnya, V. I. Alferov, A. L. Kuranov, and E. G. Sheikin. 1997. Assessment of hypersonic MHD concepts. AIAA Paper No. 972393.

3. Poggie, J., and D. Gaitonde. 2000. Magnetic control of hypersonic blunt body flow. AIAA Paper No. 2000-0452.

4. Hoffmann, K. A., H. M. Damevin, and J.-F. Dietiker. 2000. Numerical simulation of hyprsonic MHD flow. AIAA Paper No. 2000-2259.

5. Deb, P., and R. Agarwal. 2000. Numerical simulation of compressible viscous MHD flow with a bi-temperature, model for reducing supersonic drag of blunt bodies and scramjet inlets. AIAA Paper No. 2000-2419.

6. Macheret, S. O., M. N. Shneider, G. V. Candler, R. W. Moses, and J. F. Cline. 2004. Magnetohydrodynamic power generation for planetary entry vehicles. AIAA Paper No. 2004-2560.

7. Gurjanov, E. P., and P. T. Harsha. 1996. AJAX: New directions in hypersonic technology. AIAA Paper No. 1996-4609.

8. Bocharov, A. N., V. A. Bityurin, and J. Lineberry. 2005. Study of MHD interaction in hypersonic flows. 15th Conference (International) on MHD Energy Conversion Proceedings. Moscow. 399-416.

9. Bityurin, V.A., and A. N. Bocharov. 2005. MHD flow control in hypersonic flight. 15th Conference (International) on MHD Energy Conversion Proceedings. Moscow. 2:429-33.

10. Bityurin, V., A. Bocharov, D. Baranov, and S. Bychkov. 2007. Power extraction experiment with a surface MHD generator in hypersonic airflow. AIAA Paper No. 2007-3882.

11. Wan, T., G. V. Candler, S. O. Macheret, M. N. Shneider, and R. Miles. 2004. CFD modeling and simulations of MHD power generation during re-entry. AIAA Paper No. 2004-2562.

12. Bityurin, V.A., and A.N. Bocharov. 2008. MHD parachute in reentry flight. Induced electric field effects in hypersonic MHD flow. 2nd International ARA Days. Arcachon, France.

13. Bityurin, V.A., A. N. Bocharov, and N. A. Popov. 2009. Non-equilibrium effects in MHD parachute concept: Induced electric field effects. AIAA Paper No. 2009-1230.

14. Popov, N. A. 2009. Reactions of associative ionization in nitrogen with participating of excited atoms. Plasma Phys. 35(5):482-96. 
15. Aleksandrov, N.L., I. V. Kochetov, and A.L. Kuranov. 1991. Electron kinetics in weakly ionized air plasma in the cross magnetic field. Russ. J. Plasma Phys. 17(12):1446-52.

16. Erofeev, A. V., R. V. Vasil'eva, T. A. Lapushkina, and A. D. Zuev. 1997. On possibility of pre-Ionized air using in an MHD generator on aerospace plane. Symposium on Engineering Aspects of MHD. Mississippi State University. 4.3.1-4.3.12.

17. Zubkov, A. I., G. A. Tirskiy, V. A. Levin, and V. I. Sakharov. 1998. Motion of bodies in Earth's and planet's atmospheres with supersonic and hypersonic velocities under conditions of chemical conversions, heat transfer and radiation. Report No. 4507. Inst. of Mechanics of Moscow State University.

18. Andriatis, A. V., S. A. Zhluktov, and I. A. Sokolova. 1992. Transport coefficients for chemical nonequilibrium components of air mixtures. J. Math. Modeling 4(1):4464.

19. Soloviev, V.R., V. M. Krivtsov, and A. M. Konchakov. 2004. E-beam plasmas 2-D and 3-D analysis in magneto-hydrodynamic applications. AIAA Paper No. 2004183.

20. Goose, R., and G. Candler. 205. Diffusion flux modeling: Application to direct entry problems. AIAA Paper No. 2005-0389.

21. Bityurin, V.A., A. N. Bocharov, D.S. Baranov, and N. A. Popov. 2009. Progress in experimental and theoretical study of MHD parachute concept. AIAA Paper No. 2009-7318. 